\title{
MODIFYING HYPERKÄHLER MANIFOLDS WITH CIRCLE SYMMETRY*
}

\author{
ANDREW DANCER ${ }^{\dagger}$ AND ANDREW SWANN ${ }^{\ddagger}$
}

\begin{abstract}
A construction is introduced for modifying hyperkähler manifolds with triHamiltonian circle action, that in favourable situations increases the second Betti number by one. This is based on the symplectic cut construction of Lerman. In 4 or 8 dimensions the construction may be interpreted as adding a D6-brane. A number of examples are given and a generalisation to three-Sasaki geometry discussed.
\end{abstract}

Key words. Hyperkähler manifold, three-Sasaki, symplectic cut, moment map, circle action.

AMS subject classifications. Primary 53C26; Secondary 53D20, 57S25, 57N65.

1. Introduction. The symplectic cut construction of Lerman [17] has proved to be a valuable tool in the study of symplectic manifolds with Hamiltonian circle action. The purpose of this paper is to investigate analogues of this construction in quaternionic geometry, in particular for hyperkähler manifolds.

We define a modification construction for a hyperkähler manifold $M$ with triHamiltonian circle action, which involves replacing a level set of the moment map by its quotient by the circle action. The relationship between the complement of the level set in $M$ and the complement of the quotient in the new manifold $M_{\bmod }$ is more complicated than in the symplectic case. In the symplectic case one has diffeomorphisms between corresponding components, but only 'half' of the original manifold appears. For the hyperkähler modification all of $M$ is involved, but all we can say, in general, is that there is a third space which is a circle bundle over each complement.

When we perform the construction, we create a new hyperkähler submanifold of real codimension four, which is a component of the fixed set of the circle action in the modified space; this submanifold is in fact the quotient of a level set mentioned above and is the hyperkähler quotient of $M$ at the chosen level. In physical terms this can be interpreted as adding a new brane to the space.

The lowest dimensional example of this construction produces the GibbonsHawking multi-instanton spaces out of flat space. At each stage a circle level set is collapsed to a point, but the topology at long range is also changed. This can be generalised in a higher-dimensional setting to the toric hyperkähler manifolds of [3], where our construction can be interpreted as adding a new affine flat to the combinatorial data associated to the manifold.

We study how the topology changes under our construction. In particular, we prove that in the simply-connected case the second Betti number increases by one.

We conclude by briefly discussing an analogous construction for three-Sasaki spaces.

Acknowledgements. The authors thank Brian Steer and Ulrike Tillmann for useful discussions, and the Isaac Newton Institute, Cambridge, and the Erwin Schrödinger Institute, Vienna, for hospitality during the later stages of this work.

\footnotetext{
*Received October 26, 2005; accepted for publication March 13, 2006.

$\dagger$ Jesus College, Oxford, OX1 3DW, United Kingdom (dancer@maths.ox.ac.uk).

$\ddagger$ Department of Mathematics and Computer Science, University of Southern Denmark, Campusvej 55, DK-5230 Odense M, Denmark (swann@imada.sdu.dk).
} 
2. Symplectic cuts. We shall first review Lerman's symplectic cut construction [17].

Let $(M, \omega)$ be symplectic with a Hamiltonian circle action whose moment map is $\mu: M \rightarrow \mathbb{R}$. We now consider $M \times \mathbb{C}$ with the product symplectic structure and the $S^{1}$-action

$$
e^{\mathbf{i} \theta}:(m, z) \mapsto\left(e^{\mathbf{i} \theta} \cdot m, e^{-\mathbf{i} \theta} z\right) .
$$

whose moment map is

$$
\Phi:(m, z) \mapsto \mu(m)-|z|^{2} .
$$

The symplectic cut $M_{\text {cut }}=\Phi^{-1}(\varepsilon) / S^{1}$ is the symplectic quotient of $M \times \mathbb{C}$ at level $\varepsilon$. This fits into the following diagram:

$$
M \stackrel{\pi}{\leftarrow} \Phi^{-1}(\varepsilon) \stackrel{p}{\rightarrow} M_{\text {cut }} .
$$

Here $\pi: \Phi^{-1}(\varepsilon) \rightarrow M$ is projection $(m, z) \mapsto m$, and its image is $\{m \in M: \mu(m) \geqslant \varepsilon\}$. The map $p: \Phi^{-1}(\varepsilon) \rightarrow M_{\text {cut }}$ is just the quotient map for the $S^{1}$-action. If the circle action on $M$ (or, more generally, on $\Phi^{-1}(\varepsilon)$ ), is free then the fibres of $p$ are all circles. However the fibres of $\pi$ over points in its image are only circles away from the locus $\mu^{-1}(\varepsilon)$. Over $\mu^{-1}(\varepsilon)$, by contrast, $\pi$ is injective.

Notice that $\pi$ admits a section $s: m \mapsto(m,+\sqrt{\mu(m)-\varepsilon})$. This is essentially derived from the section of the map $\phi_{0}: z \mapsto|z|^{2}$, the moment map for the action on $\mathbb{C}$, which expresses $\mathbb{C}$ as a circle bundle over $\mathbb{R}_{\geqslant 0}$ with one special (point) fibre over the origin. Now $p \circ s$ gives a surjection from $\{m \in M: \mu(m) \geqslant \varepsilon\}$ onto $M_{\text {cut }}$, which is a diffeomorphism away from $\mu^{-1}(\varepsilon)$ but has circle fibres on $\mu^{-1}(\varepsilon)$.

We can rephrase this as follows. The set $\Phi^{-1}(\varepsilon)$ is the disjoint union $\Sigma_{1} \cup \Sigma_{2}$ where

$$
\begin{gathered}
\Sigma_{1}=\{(m, z): \mu(m)>\varepsilon,|z|=+\sqrt{\mu(m)-\varepsilon}\} \\
\Sigma_{2}=\{(m, 0): \mu(m)=\varepsilon\} .
\end{gathered}
$$

On $\Sigma_{1}$, each orbit of the circle action contains a unique $(m, z)$ with $z$ real and positive $(z=+\sqrt{\mu(m)-\varepsilon})$. Hence $\Sigma_{1} / S^{1}$ may be identified with $\{m: \mu(m)>\varepsilon\}$. On the other hand, $\Sigma_{2} / S^{1}$ is just the symplectic quotient $\mu^{-1}(\varepsilon) / S^{1}$.

Thus one sees that the symplectic cut $\Phi^{-1}(\varepsilon) / S^{1}$ may be viewed as coming from $\{m: \mu(m) \geqslant \varepsilon\}$ by factoring out the circle action on the boundary $\mu^{-1}(\varepsilon)$.

3. Hyperkähler modifications. To define a hyperkähler manifold we start with a Riemannian manifold $(M, g)$ and three compatible complex structures $I$, $J$ and $K$ such that $I J=K=-J I$. Compatibility means that the tensors $F_{I}(X, Y)=g(I X, Y)$, etc., are all 2-forms. One says that $M$ is hyperkähler if these three 2-forms are closed. In particular, $F_{I}, F_{J}$ and $F_{K}$ define symplectic structures on $M$.

To generalise the symplectic cut construction to hyperkähler geometry, we replace the extra factor $\mathbb{C}$ in the previous section with $\mathbb{H} \cong \mathbb{R}^{4}$. The flat metric on $\mathbb{H}$ is hyperkähler with complex structures induced by multiplication by unit quaternions on the right. Fixing $\mathbf{i}$ we will often write $\mathbb{H}=\mathbb{C}+\mathbf{j} \mathbb{C}$ with complex coordinates $(z, w)$. This carries a circle action preserving the geometry given by

$$
e^{\mathbf{i} \theta}:(z, w) \mapsto\left(e^{\mathbf{i} \theta} z, e^{-\mathbf{i} \theta} w\right) .
$$


This action is tri-Hamiltonian, meaning that it is Hamiltonian with respect to each of the three symplectic structures. We may combine the three moment maps into the single map taking values in $\mathbb{R}^{3}=\mathbb{R} \times \mathbb{C}$ :

$$
(z, w) \mapsto\left(\frac{1}{2}\left(|z|^{2}-|w|^{2}\right), \mathbf{i} z w\right) .
$$

Now let $M$ be a hyperkähler manifold with a tri-Hamiltonian circle action. Consider $M \times \mathbb{H}$ with action:

$$
e^{\mathbf{i} \theta}:(m, z, w) \mapsto\left(e^{\mathbf{i} \theta} m, e^{-\mathbf{i} \theta} z, e^{\mathbf{i} \theta} w\right) .
$$

The associated moment map $\Phi: M \times \mathbb{H} \rightarrow \mathbb{R}^{3}=\mathbb{R} \times \mathbb{C}$ is defined by

$$
\Phi:(m, z, w) \mapsto\left(\mu_{\mathbb{R}}(m)-\frac{1}{2}\left(|z|^{2}-|w|^{2}\right), \mu_{\mathbb{C}}(m)-\mathbf{i} z w\right),
$$

where $\mu=\left(\mu_{\mathbb{R}}, \mu_{\mathbb{C}}\right): M \rightarrow \mathbb{R}^{3}=\mathbb{R} \times \mathbb{C}$ is the hyperkähler moment map for the circle action on $M$.

Definition 3.1. The modification of the tri-Hamiltonian hyperkähler manifold $M$ at level $\varepsilon$ is defined to be $M_{\bmod }=\Phi^{-1}(\varepsilon) / S^{1}$, where $\varepsilon=\left(\varepsilon_{\mathbb{R}}, \varepsilon_{\mathbb{C}}\right)$ and $\Phi$ is as in equation (3.3).

The $S^{1}$-action on $M \times \mathbb{H}$ is free except at points $(m, 0,0)$ where $m$ is a point of $M_{\text {non-free }}$, the set of points of $M$ with non-trivial stabiliser. The results of [14] on the hyperkähler quotient construction, show that the modification $M_{\bmod }$ will be a smooth manifold provided we choose the level $\varepsilon$ to be outside $\mu\left(M_{\text {non-free }}\right)$.

Definition 3.2. A good modification of $M$ is the modification $M_{\bmod }$ at a level $\varepsilon$ lying in $\mu(M) \backslash \mu\left(M_{\text {non-free }}\right)$. Such an $\varepsilon$ will also be called good.

Any good modification $M_{\text {mod }}$ carries a hyperkähler structure obtained by restricting the Kähler forms of $M \times \mathbb{H}$ to the level set and descending to the quotient. This is a consequence of the general theory of hyperkähler quotients in [14]. In addition, in this case $M_{\text {mod }}$ will be complete as long as $M$ is complete.

Note that, analogously to the symplectic case, the circle action

$$
(m, z, w) \mapsto\left(e^{\mathbf{i} \psi} \cdot m, z, w\right)
$$

on $M \times \mathbb{H}$ preserves the level set of $\Phi$ and commutes with our previous $S^{1}$-action on that set. So the action (3.4) descends to a tri-Hamiltonian action on $M_{\text {mod. }}$. More specifically, writing $[m, z, w]$ for the point of $M_{\text {mod }}$ represented by $(m, z, w) \in \Phi^{-1}(\varepsilon)$, the moment map for the action on $M_{\text {mod }}$ is $[m, z, w] \mapsto \mu(m)$.

Summarising the discussion so far we have:

Proposition 3.3. Let $M$ be a hyperkähler manifold with tri-Hamiltonian circle action with moment map $\mu: M \rightarrow \mathbb{R}^{3}$. Then each good modification $M_{\bmod }$ of $M$ is a again a smooth hyperkähler manifold, of the same dimension as $M$, with triHamiltonian circle action.

Note that the circle action on $M_{\text {mod }}$ induced by (3.4) is free except when there exist $e^{\mathbf{i} \theta} \in S^{1}$ and $e^{\mathbf{i} \psi} \in S^{1} \backslash\{1\}$ with

$$
\left(e^{\mathbf{i} \psi} \cdot m, z, w\right)=\left(e^{\mathbf{i} \theta} \cdot m, e^{-\mathbf{i} \theta} z, e^{\mathbf{i} \theta} w\right) .
$$


This set is the union of the two sets

$$
\begin{gathered}
\{[m, 0,0]: \mu(m)=\varepsilon\}, \\
\left\{[m, z, w]: m \in M_{\text {non-free }}, \Phi(m, z, w)=\varepsilon\right\}
\end{gathered}
$$

so

$$
\mu\left(\left(M_{\text {mod }}\right)_{\text {non-free }}\right)=\{\varepsilon\} \cup \mu\left(M_{\text {non-free }}\right) .
$$

The modification construction may therefore be iterated, provided that we choose our level set suitably at each stage (and $\mu(M) \backslash \mu\left(M_{\text {non-free}}\right)$ is not a finite set). In particular, if the circle action on $M$ is free then we may iterate the construction provided we change the level set at each stage.

REMARK 3.4. If one chooses to work in the orbifold category then all that one requires is that the circle action be non-trivial and that the fixed-point set $M^{S^{1}}$ does not meet the level set $\mu^{-1}(\varepsilon)$.

4. Topology. Let us now analyse the structure of the modification $M_{\text {mod }}$. Recall (see [3] for example) that (3.2) is a map $\phi: \mathbb{H} \rightarrow \mathbb{R}^{3}=\mathbb{R} \times \mathbb{C}$ such that $\phi^{-1}(0,0)$ is $(0,0)$ and whose fibre over any other point is exactly a free orbit of the circle action (3.1). This is the hyperkähler analogue of the map $\phi_{0}: z \mapsto|z|^{2}$ from $\mathbb{C}$ to $\mathbb{R}$ discussed in the symplectic case. However, there are two important differences:

(i) the map $\phi$ is onto $\mathbb{R}^{3}$, whereas its symplectic analogue $\phi_{0}$ is only onto the closed halfline $\mathbb{R}_{\geqslant 0} \subset \mathbb{R}$

(ii) the map $\phi$ does not admit a section, unlike $\phi_{0}$. Indeed on spheres in $\mathbb{H}$, we see that $\phi$ is the Hopf fibration $S^{3} \rightarrow S^{2}$.

Point (i) is the reason for our choice of terminology 'modification' instead of 'cut' since the whole of $M$ contributes to the construction of $M_{\text {mod }}$ rather than a proper subset.

We have a diagram

$$
M \stackrel{\pi}{\leftarrow} N \stackrel{p}{\rightarrow} M_{\text {mod }}
$$

Here $N=\Phi^{-1}(\varepsilon)$ and as before $p$ is just the quotient map for the circle action on $N$, which has circle fibres if $S^{1}$ acts freely on $N$. Also $\pi: N \rightarrow M$ is just projection $(m, z, w) \mapsto m$. It is now onto $M$ (in contrast to the symplectic case) because of (i). So we do not remove half the manifold when we perform the hyperkähler modification.

The fibre of $\pi$ is a circle except on the set $\tilde{X}=\mu^{-1}(\varepsilon) \times\{(0,0)\}$, where $\pi$ is injective. Thus under the modification, the set $X=\mu^{-1}(\varepsilon)$ in $M$ is being replaced by its $S^{1}$-quotient $\hat{X}=X / S^{1}$, the hyperkähler quotient of $M$ at level $\varepsilon$, in $M_{\bmod }$. When $\varepsilon$ is good, this set is a component of the fixed-point set of the circle action (3.4) on $M_{\text {mod }}$, see the discussion after Proposition 3.3. Using the results of [10], we thus have:

Proposition 4.1. Suppose $M_{\bmod }$ is a good modification of $M$ at level $\varepsilon$. Then $M_{\bmod }$ contains the hyperkähler quotient of $M$ at level $\varepsilon$ as a hyperkähler submanifold of codimension 4.

The relation between the complements $M^{*}=M \backslash X$ and $M_{\text {mod }}^{*}=M_{\text {mod }} \backslash \hat{X}$ is more complicated than in the symplectic case, as (due to (ii)) the map $\pi$ does not admit a global section. What we do have is a space $N^{*}=N \backslash \tilde{X}$ which is a circle bundle over both $M^{*}$ and $M_{\text {mod }}^{*}$. 
REMARK 4.2 . If $\mu(M) \neq \mathbb{R}^{3}$ then one also has the possibility of modifying $M$ at a level $\varepsilon \notin \mu(M)$. In this situation no new fixed-points are created, but $M_{\text {mod }}$ will not be isometric to $M$ and again there will be a common circle bundle above both spaces. This situation is excluded by our definition of good modification.

4.1. Euler characteristic. As mentioned above the collapsed set $\hat{X}$ in $M_{\bmod }$ will be a component $L$ of the fixed-point set of the hyperkähler circle action (3.4) on $M_{\text {mod }}$. It has real codimension 4 in $M_{\text {mod }}$ and its normal bundle will be a nontrivial circle representation of quaternionic dimension one.

Suppose the circle action on $M_{\text {mod }}$ has no non-trivial finite isotropy groups, and that all components of $L$ are of codimension 4 . Then $M_{\text {mod }} / S^{1}$ will be a smooth manifold: the spheres in the normal bundle are copies of $S^{3}$, whose quotient by $S^{1}$ will be $S^{2}$. As this is a boundary it can therefore be filled in in the quotient, see $[12,13,1]$.

In the language of string theory such a component of $L$ is a D6-brane when the ambient space is 4- or 8-dimensional. Whenever we modify $M$, therefore, we are adding in a new brane whose position is determined by the level $\varepsilon$.

Note also that, by a standard argument, the Euler characteristic of $M_{\bmod }$ will be the sum of the Euler characteristics of the components of the fixed-point sets of the circle action. Indeed if $L$ has finitely many connected components $L_{i}$, for $i=1, \ldots, k$, and $V_{i}$ are mutually disjoint tubular neighbourhoods of $L_{i}$, then as the circle action on $M_{\text {mod }} \backslash L$ is free, we see that the intersection with $V_{i}$ has $\chi=0$, so

$$
\chi\left(M_{\text {mod }}\right)=\sum_{i=1}^{k} \chi\left(V_{i}\right)+\chi\left(M_{\text {mod }} \backslash L\right)=\sum_{i=1}^{k} \chi\left(L_{i}\right)=\chi(L) .
$$

This can sometimes yield useful information on the topology of $M_{\bmod }$.

4.2. Cohomology. Our aim is to relate the cohomology of $M$ and its good modification $M_{\text {mod }}$. In our cohomology calculations we shall take coefficients over $\mathbb{R}$ unless otherwise stated. We shall also assume that our manifolds have finite topological type.

We use the notation introduced at the beginning of this section, and in addition choose a tubular neighbourhood $U$ of $X=\mu^{-1}(\varepsilon)$ in $M$. Noting that $\tilde{X}=\pi^{-1}(X)$, we write $\tilde{U}=\pi^{-1}(U)$ and put $U_{\bmod }=p(\tilde{U})$. Again $p(\tilde{X})=\hat{X}$ and $U_{\bmod }$ is a neighbourhood of $\hat{X}$ in $M_{\bmod }$. As above, a star ${ }^{*}$ on a set will denote the complement of the corresponding set associated to $X$, e.g., $U_{\bmod }^{*}=U_{\bmod } \backslash \hat{X}$.

Observe that in the diagram (4.1), $N^{*}$ is the total space of circle fibrations over both $M^{*}$ and $M_{\bmod }^{*}$. We denote the Euler classes by $e$ and $e^{\prime}$, respectively. Similarly, we have that $\tilde{U}^{*}$ is the total space of circle fibrations over $U^{*}$ and $U_{\text {mod }}^{*}$.

We note that the normal bundle of $X$ in $M$ is trivial. In fact if $\xi$ denotes the Killing field for the circle action on $X$, then $I \xi, J \xi, K \xi$ give an explicit trivialisation. Hence we may take $U^{*}$ to be homotopic to $S^{2} \times X$.

Lemma 4.3. Suppose $M$ is simply connected. Then $M_{\bmod }$ is also simply connected and

$$
b_{2}\left(M^{*}\right)=b_{2}\left(M_{\bmod }^{*}\right)=b_{2}\left(M_{\bmod }\right) .
$$

Proof. As $X$ has codimension 3 in $M$, we deduce that $M^{*}$ is also simply connected so $H^{1}\left(M^{*}, \mathbb{Z}\right)=0$ and $H^{2}\left(M^{*}, \mathbb{Z}\right)$ is torsion-free, by the Universal Coefficient Theorem. 
Applying the Gysin sequence to the fibration $S^{1} \rightarrow N^{*} \stackrel{\pi}{\rightarrow} M^{*}$ we obtain:

$$
0 \rightarrow H^{1}\left(N^{*}\right) \stackrel{\pi_{*}}{\rightarrow} H^{0}\left(M^{*}\right) \stackrel{\wedge e}{\rightarrow} H^{2}\left(M^{*}\right) \stackrel{\pi^{*}}{\rightarrow} H^{2}\left(N^{*}\right) \stackrel{\pi_{*}}{\rightarrow} H^{1}\left(M^{*}\right)=0 .
$$

As remarked after Proposition 4.1, $\pi$ admits no section, so the Euler class $e$ is non-zero. We deduce that $H^{0}\left(M^{*}\right)$ injects into $H^{2}\left(M^{*}\right)$, that $H^{1}\left(N^{*}\right)=0$ and that

$$
b_{2}\left(M^{*}\right)=b_{2}\left(N^{*}\right)+1 .
$$

The long exact homotopy sequence from $S^{1} \rightarrow N^{*} \stackrel{p}{\rightarrow} M^{*}$ yields:

$$
1 \rightarrow \pi_{2}\left(N^{*}\right) \rightarrow \pi_{2}\left(M^{*}\right) \rightarrow \mathbb{Z} \rightarrow \pi_{1}\left(N^{*}\right) \rightarrow 1 ;
$$

hence $\pi_{1}\left(N^{*}\right)$ is a homomorphic image of $\mathbb{Z}$. As $H^{1}\left(N^{*}\right)=0$, we deduce that $\pi_{1}\left(N^{*}\right)$ is trivial or $\mathbb{Z} / m \mathbb{Z}$ for some $m$. In the latter case the universal cover $P$ of $N^{*}$ is a principal circle bundle $P \rightarrow M^{*}$ such that $P^{m}=N^{*}$. However, the set $U \subset M$ is diffeomorphic to the normal bundle of $X$ in $M$, and over a fibre of this normal bundle the restriction of $N^{*}$ is homotopic to the Hopf fibration, so $N^{*}$ admits no such $m$ th root $P$. We conclude that $N^{*}$ is simply connected.

The long exact homotopy sequence from $S^{1} \rightarrow N^{*} \rightarrow M_{\text {mod }}^{*}$ now gives:

$$
1 \rightarrow \pi_{2}\left(N^{*}\right) \rightarrow \pi_{2}\left(M_{\mathrm{mod}}^{*}\right) \rightarrow \mathbb{Z} \rightarrow \pi_{1}\left(N^{*}\right)=1 \rightarrow \pi_{1}\left(M_{\mathrm{mod}}^{*}\right) \rightarrow 1
$$

so $\pi_{1}\left(M_{\mathrm{mod}}^{*}\right)$ is trivial.

The Gysin sequence from $S^{1} \rightarrow N^{*} \stackrel{p}{\rightarrow} M_{\text {mod }}^{*}$ gives:

$$
0 \rightarrow H^{0}\left(M_{\text {mod }}^{*}\right) \stackrel{\wedge e^{\prime}}{\rightarrow} H^{2}\left(M_{\text {mod }}^{*}\right) \stackrel{p^{*}}{\rightarrow} H^{2}\left(N^{*}\right) \stackrel{p_{*}}{\rightarrow} H^{1}\left(M_{\text {mod }}^{*}\right)=0 .
$$

We deduce that $e^{\prime} \neq 0$ and

$$
b_{2}\left(M_{\text {mod }}^{*}\right)=b_{2}\left(N^{*}\right)+1
$$

Now $\hat{X}$ is codimension 4 in $M_{\text {mod }}$, so $\pi_{1}\left(M_{\text {mod }}\right)=\pi_{1}\left(M_{\text {mod }}^{*}\right)=1$ and $\pi_{2}\left(M_{\text {mod }}\right)=$ $\pi_{2}\left(M_{\text {mod }}^{*}\right)$. By Hurewicz $H^{2}\left(M_{\text {mod }}, \mathbb{Z}\right)=H^{2}\left(M_{\text {mod }}^{*}, \mathbb{Z}\right)$ and the required result follows from (4.2) and (4.3).

THEOREM 4.4. Suppose $M$ is a hyperkähler manifold with tri-Hamiltonian circle action and finite topological type. Let $M_{\bmod }$ be a good modification of $M$ at a level $\varepsilon$ such that $X=\mu^{-1}(\varepsilon)$ has finite topological type. If $M$ is simply connected, then

$$
b_{2}\left(M_{\text {mod }}\right)=b_{2}(M)+1 .
$$

Proof. By the Lemma, we need to compare $b_{2}\left(M^{*}\right)$ and $b_{2}(M)$.

We have a Thom-Gysin sequence

$$
\cdots \rightarrow H^{i}(M) \rightarrow H^{i}\left(M^{*}\right) \rightarrow H^{i-2}(X) \rightarrow H^{i+1}(M) \rightarrow \cdots
$$

This can be obtained by applying Mayer-Vietoris to $M=M^{*} \cup U$. Recalling that $U$ is homotopic to $X$ and that $M^{*} \cap U=U^{*}$ is homotopic to $S^{2} \times X$, we obtain:

$$
\cdots \rightarrow H^{i}(M) \rightarrow H^{i}\left(M^{*}\right) \oplus H^{i}(X) \rightarrow H^{i-2}(X) \oplus H^{i}(X) \rightarrow H^{i+1}(M) \rightarrow \cdots,
$$


where the $H^{i-2}(X) \oplus H^{i}(X)$ term is the Künneth decomposition of $H^{i}\left(S^{2} \times X\right)$. Now if $*$ is a basepoint in $S^{2}$ we can consider $X \stackrel{\kappa}{\rightarrow} S^{2} \times X \stackrel{\iota}{\rightarrow} \mathbb{R}^{3} \times X \stackrel{\pi}{\rightarrow} X$, where $\kappa: x \mapsto$ $(*, x)$, the map $\iota$ is inclusion and $\pi$ is projection. As $\pi \iota \kappa$ is the identity and $\pi^{*}$ is an isomorphism on cohomology, we deduce $\iota^{*}$ maps $H^{i}\left(\mathbb{R}^{3} \times X\right)=H^{i}(U)=H^{i}(X)$ isomorphically onto the $H^{i}(X)$-part of $H^{i}\left(S^{2} \times X\right)$. This yields the sequence (4.4).

As we are assuming $M$ is simply connected the sequence (4.4) begins

$$
0 \rightarrow H^{2}(M) \rightarrow H^{2}\left(M^{*}\right) \rightarrow \mathbb{R} \rightarrow H^{3}(M) \rightarrow H^{3}\left(M^{*}\right) \rightarrow \cdots
$$

The map from $\mathbb{R}=H^{0}(X) \rightarrow H^{3}(M)$ is the Thom isomorphism for the normal bundle of $X$ followed by extension by zero. So the image of $1 \in H^{0}(X)$ is the closed Poincaré dual $\eta_{X}$ of $X$ in $H^{3}(M)$, [4, eqn. (5.13), p. 51]. However, the operation of taking the Poincaré dual is natural with respect to maps in the sense that $\eta_{f^{-1}(S)}=f^{*} \eta_{S}$ if $S$ is transverse to $f$. This gives

$$
\eta_{X}=\eta_{\mu^{-1}(\varepsilon)}=\mu^{*} \eta_{\{\varepsilon\}},
$$

since $\varepsilon$ is a good and so a regular value of $\mu$. Now $\eta_{\{\varepsilon\}}$ is zero as it lives in $H^{3}\left(\mathbb{R}^{3}\right)=0$, so $\eta_{X}=0 \in H^{3}(M)$ and the sequence (4.5) splits after $\mathbb{R}$. Thus the desired conclusion on the second Betti numbers now follows. $\square$

\section{Examples.}

5.1. Multi-instanton metrics. Let $M=\mathbb{H}$ with circle action $q \mapsto e^{\mathbf{i} t} q$. The associated moment map $\mu: M \rightarrow \mathbb{R}^{3}$ has circle fibres, except for the point fibre over the origin. Modifying at a non-zero level, we obtain a new hyperkähler 4-manifold with a hyperkähler circle action. One of the level sets of $\mu$ has been replaced by its quotient by the circle action, that is, it has been shrunk to a point. So $M_{\bmod }$ maps to $\mathbb{R}^{3}$ but now has two point fibres. This is the Eguchi-Hanson space, which has $b_{2}=1$. Repeating the construction gives the multi-instanton series of Gibbons \& Hawking [11]. The topology of these spaces is generated by a chain of $k-1$ twospheres, where $k$ is the number of point fibres. Each time we perform the construction, therefore, the second Betti number increases by one.

5.2. Taub-NUT metrics. Let us take $M$ to be $\mathbb{R}^{3} \times S^{1}=\mathbb{H} / \mathbb{Z}$ with the flat hyperkähler structure induced from that on $\mathbb{H}$. The free circle action has a moment map which is just projection onto the $\mathbb{R}^{3}$ factor. Applying the modification construction shrinks one of the fibres to a point, and we obtain the Taub-NUT space which is topologically $\mathbb{H}$ but has a non-flat metric. Repeating the construction gives the multi-Taub-NUT series which are topologically, but not metrically, the same as the multi-instanton series.

5.3. Hyperkähler toric manifolds. A generalisation of Example $\S 5.1$ is provided by the toric hyperkähler manifolds of [3]. These arise as hyperkähler quotients of $\mathbb{H}^{d}$ by a sub-torus $\mathcal{N}$ of $\mathbb{T}^{d}$. We choose $\mathcal{N}$ by taking its Lie algebra $\mathfrak{n}$ to be the kernel of a surjective linear map $\beta: \mathbb{R}^{d} \rightarrow \mathbb{R}^{n}$ given by

$$
\beta: e_{k} \longmapsto u_{k},
$$

with $u_{i} \in \mathbb{Z}^{n}$ (here $e_{1}, \ldots, e_{d}$ are the standard basis vectors for $\mathbb{R}^{d}$ ). So $\mathcal{N}$ is determined by a choice of $d$ vectors $u_{1}, \ldots, u_{d}$ spanning $\mathbb{R}^{n}$.

The hyperkähler quotient $M$ has dimension $4 n$, and $n=d-\operatorname{dim} \mathcal{N}$. Much of the geometry of $M$ is determined by the flats $H_{k}, k=1, \ldots, d$ which are codimension 3 
affine subspaces of $\mathbb{R}^{3 n}$ defined by equations:

$$
\left\{y \in \mathbb{R}^{n} \otimes \mathbb{R}^{3}:\left\langle y, u_{k}\right\rangle=\lambda_{k}\right\},
$$

where the $\lambda_{k}=\left(\lambda_{k}^{1}, \lambda_{k}^{2}, \lambda_{k}^{3}\right) \in \mathbb{R}^{3}$. The projections of $\sum_{k=1}^{d} \lambda_{k}^{j} e_{k}, j=1,2,3$, to $\mathfrak{n}^{*}$ give the choice of level set involved in defining the hyperkähler quotient $M$.

Now $M$ admits a hyperkähler action of $\mathbb{T}^{n}=\mathbb{T}^{d} / \mathcal{N}$. The moment map $\phi: M \rightarrow$ $\mathbb{R}^{3 n}$ for this action is surjective and its fibres over generic points are copies of $\mathbb{T}^{n}$. However the fibres are lower-dimensional tori over points lying in the union of the flats $H_{k}$. More precisely, the $\mathbb{T}^{n}$-stabiliser of a point in $M$ lying over $y$ has Lie algebra spanned by those vectors $u_{k}$ such that $y \in H_{k}$, see [3, §3]. Also, $M$ has at worst orbifold singularities if and only if no $n+1$ flats meet.

The modification $M_{\text {mod }}$ may also be viewed as a toric hyperkähler manifold which is now a quotient of $\mathbb{H}^{d+1}$ by a torus $\hat{\mathcal{N}}$ with Lie algebra $\hat{\mathfrak{n}}$. We can view $\hat{\mathfrak{n}}$ as the kernel of a map $\hat{\beta}: \mathbb{R}^{d+1} \rightarrow \mathbb{R}^{n}$, where $\hat{\beta}$ is defined as in (5.1), except that we now introduce an additional vector $u_{d+1}=\sum_{i=1}^{d} \xi_{i} u_{i}$. So $\hat{\mathfrak{n}}$ is an extension of $\mathfrak{n}$ by the 1-dimensional algebra $\langle\xi\rangle$. (We can regard $\xi$ as the generator of the circle action on $M$ by which we choose to perform the modification).

In terms of the configuration of flats, forming $M_{\text {mod }}$ from $M$ corresponds therefore to adding one new flat $H_{d+1}$.

Note that the multi-instanton spaces of Example $\S 5.1$ are just the $n=1$ case of this discussion. Here the flats $H_{k}$ are just points in $\mathbb{R}^{3}$.

In [3, §6], the topology of $M$ was examined in terms of the combinatorial data of the flats. We considered the hyperplanes $H_{k}^{1}$ in $\mathbb{R}^{n}$ defined by $\left\langle x, u_{k}\right\rangle=\lambda_{k}^{1}$. These divide up $\mathbb{R}^{n}$ into a union of polyhedra, and we formed the bounded polyhedral complex $\mathcal{C}$ consisting of the bounded polyhedra in this collection and their faces.

Theorem 6.5 of [3] showed that $M$ is simply connected with Poincaré polynomial

$$
P_{t}(M)=\sum_{k=0}^{n} d_{k}\left(t^{2}-1\right)^{k}
$$

where $d_{k}$ is the number of $k$-dimensional elements of $\mathcal{C}$. In particular, the odddimensional cohomology vanishes. (Theorem 6.5 is in fact still true if $M$ is allowed to have orbifold singularities $)$. It follows from the discussion of $\S 4.2$ that $b_{2}\left(M_{\text {mod }}\right)=$ $b_{2}(M)+1$.

As an example we can consider the Calabi space $M=T^{*} \mathbb{P}^{2}$, which corresponds to taking $d=3, n=2, u_{1}=e_{1}, u_{2}=e_{2}$ and $u_{3}=-\left(e_{1}+e_{2}\right)$. The complex $\mathcal{C}$ therefore consists of a right triangle and its faces. We have a $\mathbb{T}^{2}$-action on $M$ and can choose any circle subgroup of $\mathbb{T}^{2}$ in forming the modified space $M_{\text {mod }}$.

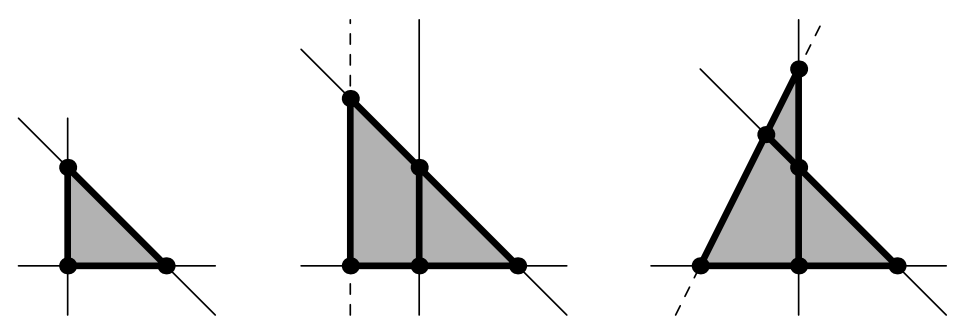

Figure 5.1. $T^{*} \mathbb{P}^{2}$ (left) and two of its modifications. 
Forming $M_{\text {mod }}$ will involve adding a new hyperplane; depending on the choice of hyperplane (i.e., the choice of circle action) we can arrange for $\left(d_{1}, d_{2}\right)$ of $M_{\bmod }$ to equal $(6,2)$ or $(8,3)$, see Figure 5.1. (For $M_{\text {mod }}$ to be an orbifold we require that no three hyperplanes meet).

The Betti numbers of a toric hyperkähler orbifold with $n=2$ are, from (5.2), given by $b_{2}=d_{1}-2 d_{2}$ and $b_{4}=d_{2}$. Note that for $M$ we have $\left(d_{1}, d_{2}\right)=(3,1)$ so $b_{2}=b_{4}=1$, as we know from the description of $M$ as $T^{*} \mathbb{P}^{2}$.

Both the possibilities for $M_{\text {mod }}$ mentioned above yield $b_{2}=2$ but $b_{4}$ can be 2 or 3 depending on the choice of circle action.

The quadratic homogeneity of the moment map involved in forming toric hyperkähler quotients means that we can apply the arguments for asymptotics used by Kronheimer [16] in four dimensions. Radially scaling by $r$ in $\mathbb{H}^{d}$ corresponds to staying instead at the same distance from 0 in $\mathbb{H}^{d}$ and scaling $\lambda$ by $r^{-2}$. It follows that the toric hyperkähler quotients are asymptotically conical in generic directions. The base of the cone is an open set in the three-Sasaki space corresponding to $\lambda=0$, cf. $\S 6$. However there may be bad directions where this fails, corresponding to singularities of the three-Sasaki quotient (cf. Bielawski [2]).

Forming the modification, therefore, preserves the generic asymptotically conical nature of the manifold, but will in general alter the base of the cone.

More generally, for general hyperkähler manifolds, we conjecture that generic asymptotically conical or asymptotically locally conical (asymptotic to a constant circle over a cone) behaviour will be preserved under modification, but with the base of the cone being changed, and possibly with bad non-generic directions being created.

5.4. Gauge theory quotients. We shall now consider some spaces arising from infinite-dimensional gauge theoretic constructions. In contrast to the preceding examples, these spaces may have odd-dimensional cohomology (in particular non-zero $\left.b_{3}\right)$.

Recall from [15] that the cotangent bundle $T^{*} G_{\mathbb{C}}$ of the complexification of a compact Lie group $G$ admits a hyperkähler structure. This is obtained by identify$\operatorname{ing} T^{*} G_{\mathbb{C}}$ with the space of $\mathfrak{g}$-valued solutions to Nahm's equations smooth on $[0,1]$, modulo gauge transformations that are the identity at $t=0,1$.

These spaces were analysed by the authors in [9]. They admit two commuting actions of $G$ preserving the hyperkähler structure, corresponding to gauge transformations that are the identity at one endpoint but not necessarily at both. It follows from [9, Proposition 4] that these actions are free, as that Proposition constructed equivariant diffeomorphisms between $T^{*} G_{\mathbb{C}}$ and an open set in $G \times\left(\mathfrak{g}^{*}\right)^{3}$ containing $G \times(0,0,0)$, where $G$ acts by left or right translations on the factor $G$ in the image.

We therefore have a large supply of free hyperkähler circle actions on $T^{*} G_{\mathbb{C}}$. We can perform the modification construction repeatedly on $T^{*} G_{\mathbb{C}}$, giving new families of hyperkähler manifolds of dimension $4 \operatorname{dim} G$. Using a single circle one obtains for example manifolds with $G \times N\left(S^{1}\right)$-symmetry containing, where $N\left(S^{1}\right)$ is the normaliser of the chosen circle $S^{1}$ in $G$. Some of these examples will contain $T^{*}\left(G_{\mathbb{C}} / \mathbb{C}^{*}\right)$ in the fixed set of the circle action. Repeatedly modifying with respect to factors of a maximal torus $\mathbb{T}^{n}$, one can also obtain families of $G \times \mathbb{T}^{n}$-invariant complete hyperkähler metrics.

Other examples of hyperkähler spaces with free group actions may be obtained by considering moduli spaces of Nahm data defined on sets of intervals, where the Nahm matrices are non-singular at some subset of endpoints. An example is the 
hyperkähler 8-manifold $M$ with free $S U(2)$-action studied in $[7,8]$. This space is homeomorphic to $S U(2) \times \mathbb{R}^{5}$. The hyperkähler quotients $\mu^{-1}(\varepsilon) / S^{1}$ of $M$ by a circle subgroup of $S U(2)$ all have the homotopy type of the double cover of the AtiyahHitchin manifold, which corresponds to taking $\varepsilon=0$. This non-compact 4-manifold retracts onto $S^{2}$. We deduce from $\S 4.1$ that the corresponding modification $M_{\text {mod }}$ has Euler characteristic equal to 2 .

5.5. Toric modifications. In the symplectic case, Burns, Guillemin \& Lerman [6] have generalised the cut construction by considering symplectic manifolds $M$ with a $\mathbb{T}^{n}$ action and moment map $\mu$. They then take a toric variety $X$ of complex dimension $n$ with a $\mathbb{T}^{n}$-action and associated moment map $\psi: X \rightarrow \Delta$, where $\Delta$ is a polytope in $\mathbb{R}^{n}$, and consider the product $M \times X$ with the anti-diagonal $\mathbb{T}^{n}$-action and associated moment map $\mu-\psi$. The symplectic quotient is then called the symplectic cut of $M$ by the toric variety $X$. One can think of the cut as being obtained by removing the complement of $\mu^{-1}(\Delta+\varepsilon)$ and factoring out on the boundary the actions of the torus stabilisers corresponding to faces of $\Delta$.

We may perform an analogous construction in the hyperkähler case, taking $X$ to be one of the toric hyperkähler manifolds mentioned in Example $\S 5.3$ with quaternionic dimension $n$. As mentioned above the hyperkähler moment map $\phi: X \rightarrow \mathbb{R}^{3 n}$ is surjective, and its fibres are generically $\mathbb{T}^{n}$ but become lower-dimensional tori on the intersections $I_{j}$ of the flats $H_{k}$. At points of $X$ in the pre-image of such intersections, the $\mathbb{T}^{n}$-action has non-trivial torus stabilisers, see [3, §3].

Forming the generalised hyperkähler modification $\left(M_{\mathrm{mod}}\right)_{X}$ therefore involves factoring out the sets $\mu^{-1}\left(I_{j}+\varepsilon\right)$ by the corresponding torus stabilisers. The complement of these sets in $M$ is not necessarily diffeomorphic to the complement in $\left(M_{\bmod }\right)_{X}$ of the quotients of these sets; as in the original construction, all we can say is that there is a third space which is a $\mathbb{T}^{n}$-bundle over each complement.

6. Modifying three-Sasaki structures. A three-Sasaki manifold $(\mathcal{S}, g)$ is best described by the property that the cone $\left(C(\mathcal{S})=\mathbb{R}_{>0} \times \mathcal{S}, d t^{2}+t^{2} g\right)$ is hyperkähler. Three-Sasaki manifolds are of dimension $4 n+3$ and provide many interesting examples of compact Einstein manifolds of positive scalar curvature; see the survey [5] and more recent papers by the same authors.

If $\mathcal{S}$ admits a circle action, then this provides a tri-Hamiltonian symmetry of the cone $C(\mathcal{S})$. On $C(\mathcal{S})$ there is a unique choice of hyperkähler moment map $\mu$ that is homogeneous with respect to scaling in the $t$ variable. The restriction $\mu_{\mathcal{S}}$ of $\mu$ to $\mathcal{S}$ at $t=1$ may then be used to define a quotient construction in the three-Sasaki category provided one reduces at the level 0 .

To modify $\mathcal{S}$ with respect to the circle action, consider the hyperkähler modification of $C(\mathcal{S})$ at level 0 . This is the quotient of the set

$$
\left\{(t, s, z, w) \in \mathbb{R}_{>0} \times \mathcal{S} \times \mathbb{C} \times \mathbb{C}: t^{2} \mu_{\mathcal{S}}(s)=\left(\frac{1}{2}\left(|z|^{2}-|w|^{2}\right), \mathbf{i} z w\right)\right.
$$

by the action

$$
e^{\mathbf{i} \theta}:(t, s, z, w) \mapsto\left(t, e^{\mathbf{i} \theta} \cdot s, e^{-\mathbf{i} \theta} z, e^{\mathbf{i} \theta} w\right) .
$$

Since the defining equations in (6.1) are homogeneous with respect to the scaling $(t, s, z, w) \mapsto(\lambda t, s, \lambda z, \lambda w)$ the quotient space is a hyperkähler cone of a three-Sasaki space $\mathcal{S}_{\text {mod }}$ that we call the modification of $\mathcal{S}$.

More concretely, $\mathcal{S}_{\text {mod }}$ is the quotient of the set of points in (6.1) satisfying

$$
t^{2}+|z|^{2}+|w|^{2}=1
$$


by the action (6.2). This is a smooth three-Sasaki manifold of the same dimension as $\mathcal{S}$ provided the circle action is free on $\mu_{\mathcal{S}}^{-1}(0) \subset \mathcal{S}$.

Proposition 6.1. Let $\mathcal{S}$ be a compact three-Sasaki manifold with circle action that is free on $\mu_{\mathcal{S}}^{-1}(0)$. The three-Sasaki modification $\mathcal{S}_{\bmod }$ is also compact and contains a copy of the three-Sasaki quotient of $\mathcal{S}$.

Proof. It is sufficient to show that $t$ is bounded away from zero on the intersection of (6.3) with (6.1).

As $\mathcal{S}$ is compact, there is a $K>0$ such that $\left\|\mu_{\mathcal{S}}(s)\right\| \leqslant K$ for all $s \in \mathcal{S}$. Since $\left(\frac{1}{2}\left(|z|^{2}-|w|^{2}\right), \mathbf{i} z w\right)$ has length $\frac{1}{2}\left(|z|^{2}+|w|^{2}\right)$, we see that

$$
t^{2} K \geqslant\left\|t^{2} \mu_{\mathcal{S}}(s)\right\|=\frac{1}{2}\left(1-t^{2}\right)
$$

Thus $t^{2} \geqslant 1 /(1+2 K)$, as required.

The three-Sasaki quotient of $\mathcal{S}$ arises as the image of the points where $z=0=w$. $\mathbf{\square}$

Simple examples of this construction are provided by taking $\mathcal{S}$ to be a round sphere $S^{4 n-1} \subset \mathbb{H}^{n}$ with the diagonal circle action. The modification is then the three-Sasaki quotient of the sphere $S^{4 n+3}$ by the circle with weights $(1, \ldots, 1,-1)$, which is shown to be smooth in [5]. More generally, if we consider the circle action on $S^{4 n-1}$ with pairwise co-prime non-zero weights $\left(p_{1}, \ldots, p_{n}\right)$, then the modification is the smooth three-Sasaki quotient of $S^{4 n+3}$ by the circle of weights $\left(p_{1}, \ldots, p_{n},-1\right)$. The cohomology calculations of Bielawski [2] again show that for toric three-Sasaki orbifolds the modification increases the second Betti number by one.

\section{REFERENCES}

[1] M. F. Atiyah and E. Witten, $M$-theory dynamics on a manifold of $G_{2}$ holonomy, Adv. Theor. Math. Phys., 6:1 (2002), pp. 1-106.

[2] R. Bielawski, Betti numbers of 3-Sasakian quotients of spheres by tori, Bull. London Math. Soc., 29 (1997), pp. 731-736.

[3] R. Bielawski and A. S. Dancer, The geometry and topology of toric hyperkähler manifolds, Comm. Anal. Geom., 8:4 (2000), pp. 727-760.

[4] R. Bott And L. W. Tu, Differential forms in algebraic topology, Graduate Texts in Mathematics, vol. 82, Springer-Verlag, New York, 1982.

[5] C. P. Boyer and K. Galicki, 3-Sasakian manifolds, Surveys in differential geometry: essays on Einstein manifolds, Surv. Differ. Geom., VI, Int. Press, Boston, MA, 1999, pp. 123-184.

[6] D. Burns, V. Guillemin, And E. Lerman, Kähler cuts, December 2002, eprint arXiv: math.DG/0212062.

[7] A. S. Dancer, Nahm's equations and hyperkähler geometry, Comm. Math. Phys., 158 (1993), pp. 545-568.

[8] — A family of hyperkähler manifolds, Quart. J. Math. Oxford, 45 (1994), pp. 463-478.

[9] A. S. Dancer and A. F. Swann, HyperKähler metrics associated to compact Lie groups, Math. Proc. Camb. Phil. Soc., 120 (1996), pp. 61-69.

[10] - The geometry of singular quaternionic Kähler quotients, International J. Math., 8 (1997), pp. 595-610.

[11] G. W. Gibbons and S. W. Hawking, Gravitational multi-instantons, Phys. Lett., B78 (1978), pp. $430-432$.

[12] S. Gukov, M-theory on manifolds with exceptional holonomy, Strings and geometry, Clay Math. Proc., vol. 3, Amer. Math. Soc., Providence, RI, 2004, pp. 127-158.

[13] S. Gukov and J. Sparks, M-theory on Spin(7) manifolds, Nuclear Phys. B, 625:1-2 (2002), pp. 3-69.

[14] N. J. Hitchin, A. Karlhede, U. Lindström, and M. RočeK, HyperKähler metrics and supersymmetry, Comm. Math. Phys., 108 (1987), pp. 535-589.

[15] P. B. Kronheimer, A hyperKähler structure on the cotangent bundle of a complex Lie group, preprint, MSRI, 1986, eprint arXiv:math.DG/0409253. 
[16] The construction of ALE spaces as hyper-Kähler quotients, J. Differential Geom., 29 (1989), pp. 665-683.

[17] E. Lerman, Symplectic cuts, Math. Res. Lett., 2:3 (1995), pp. 247-258. 